\title{
A new application of boundary integral behaviors of harmonic functions to the least harmonic majorant
}

\author{
Minghua Han ${ }^{1}$, Jianguo Sun ${ }^{2}$ and Gaoying Xue $\mathrm{e}^{3^{*}}$
}

\section{"Correspondence:}

3246346184@qq.com

${ }^{3}$ School of Mechanical Engineering,

Taizhou University, Taizhou, 317000,

China

Full list of author information is

available at the end of the article

\begin{abstract}
Our main aim in this paper is to obtain a new type of bo'nda integral behaviors of harmonic functions in a smooth cone. As an applicati the lea. armonic majorant of a nonnegative subharmonic function is also give 1 .
\end{abstract}

Keywords: boundary integral behavior; subbarn nic funs, ion; harmonic majorant

\section{Introduction}

Let $B(P, R)$ denote the open ball with center at $P$ and radius $R$ in $\mathbf{R}^{n}$, where $\mathbf{R}^{n}$ is the $n$ dimensional Euclidean spac $P \in$ and $R>0$. Let $B(P)$ denote the neighborhood of $P$ and $S_{R}=B(O, R)$ for simplic $\mathrm{Tr} e$ unit sphere and the upper half unit sphere in $\mathbf{R}^{n}$ are denoted by $\mathbf{S}_{1}$ ar $\mathbf{S}_{1}^{+}$, respe, avely. For simplicity, a point $(1, \Theta)$ on $\mathbf{S}_{1}$ and the set $\{\Theta ;(1, \Theta) \in \Gamma\}$ for 2 se $\Gamma,-\mathbf{S}_{1}$, are often identified with $\Theta$ and $\Gamma$, respectively. Let $\Lambda \times \Gamma$ denote t. et $\left\{\left(r, \Theta, \in \mathbf{R}^{n} ; r \in \Lambda,(1, \Theta) \in \Gamma\right\}\right.$, where $\Lambda \subset \mathbf{R}_{+}$and $\Gamma \subset \mathbf{S}_{1}$. We denote the set $\left.\mathbf{R}_{+}>\mathbf{S}_{1}^{+}=\left\{1-x_{v}\right) \in \mathbf{R}^{n} ; x_{n}>0\right\}$ by $\mathbf{T}_{n}$, which is called the half space.

We s! all also write $h_{1} \approx h_{2}$ for two positive functions $h_{1}$ and $h_{2}$ if and only if there exists a sitive constant $a$ such that $a^{-1} h_{1} \leq h_{2} \leq a h_{1}$. We denote $\max \{u(r, \Theta), 0\}$ and $\mathrm{n} \times \mathbf{x}\left\{-u(r, 0,0\}\right.$ by $u^{+}(r, \Theta)$ and $u^{-}(r, \Theta)$, respectively.

The $\quad \mathbf{R}_{+} \times \Gamma$ in $\mathbf{R}^{n}$ is called a cone. We denote it by $\mathfrak{C}_{n}(\Gamma)$, where $\Gamma \subset \mathbf{S}_{1}$. The sets $I \times \Gamma$ and $I \times \partial \Gamma$ with an interval on $\mathbf{R}$ are denoted by $\mathfrak{C}_{n}(\Gamma ; I)$ and $\mathfrak{S}_{n}(\Gamma ; I)$, respectively. wr denote $\mathfrak{C}_{n}(\Gamma) \cap S_{R}$ and $\mathfrak{S}_{n}(\Gamma ;(0,+\infty))$ by $\mathfrak{S}_{n}(\Gamma ; R)$ and $\mathfrak{S}_{n}(\Gamma)$, respectively.

Furthermore, we denote by $d \sigma$ (resp. $\left.d S_{R}\right)$ the $(n-1)$-dimensional volume elements induced by the Euclidean metric on $\partial \mathfrak{C}_{n}(\Gamma)$ (resp. $S_{R}$ ) and by $d w$ the elements of the Euclidean volume in $\mathbf{R}^{n}$.

It is known (see, e.g., [1], p.41) that

$$
\begin{aligned}
& \Delta^{*} \varphi(\Theta)+\lambda \varphi(\Theta)=0 \quad \text { in } \Gamma, \\
& \varphi(\Theta)=0 \quad \text { on } \partial \Gamma,
\end{aligned}
$$

where $\Delta^{*}$ is the Laplace-Beltrami operator. We denote the least positive eigenvalue of this boundary value problem (1.1) by $\lambda$ and the normalized positive eigenfunction corresponding to $\lambda$ by $\varphi(\Theta), \int_{\Gamma} \varphi^{2}(\Theta) d S_{1}=1$. 
We remark that the function $r^{\aleph^{ \pm}} \varphi(\Theta)$ is harmonic in $\mathfrak{C}_{n}(\Gamma)$, belongs to the class $C^{2}\left(\mathfrak{C}_{n}(\Gamma) \backslash\{O\}\right)$ and vanishes on $\mathfrak{S}_{n}(\Gamma)$, where

$$
2 \aleph^{ \pm}=-n+2 \pm \sqrt{(n-2)^{2}+4 \lambda}
$$

For simplicity we shall write $\chi$ instead of $\aleph^{+}-\aleph^{-}$.

For simplicity we shall assume that the boundary of the domain $\Gamma$ is twice continuously differentiable, $\varphi \in C^{2}(\bar{\Gamma})$ and $\frac{\partial \varphi}{\partial n}>0$ on $\partial \Gamma$. Then (see [2], pp.7-8)

$$
\operatorname{dist}(\Theta, \partial \Gamma) \approx \varphi(\Theta)
$$

where $\Theta \in \Gamma$.

Let $\delta(P)=\operatorname{dist}\left(P, \partial \mathfrak{C}_{n}(\Gamma)\right)$, we have

$$
\varphi(\Theta) \approx \delta(P)
$$

for any $P=(1, \Theta) \in \Gamma$ (see $[3,4])$.

Let $u(r, \Theta)$ be a function on $\mathfrak{C}_{n}(\Gamma)$. For any given $r \in \mathbf{l}$ the inte, al

$$
\int_{\Gamma} u(r, \Theta) \varphi(\Theta) d S_{1}
$$

is denoted by $\mathcal{N}_{u}(r)$ when it exists. The nite infi, ite limit

$$
\lim _{r \rightarrow \infty} r^{-\aleph^{+}} \mathcal{N}_{u}(r)
$$

is denoted by $u_{u}$ when it s vists.

Remark 1 A function $t)$ on $(0) \infty)$ is $\mathbb{A}_{d_{1}, d_{2}}$-convex if and only if $g(t) t^{d_{2}}$ is a convex function of $t^{d}\left(d=d_{1}+d_{2}\right)$ on,$\left.-\cup\right)$ or, equivalently, if and only if $g(t) t^{-d_{1}}$ is a convex function of $t^{-d}$ on $(0, \infty)$.

Rema $2 f(r)$ is $\mathbb{A}_{\aleph^{+}, \gamma-1}$-convex on $(0, \infty)$, where $u$ is a subharmonic function on $\mathfrak{C}_{n}(\Gamma)$ surit that

$$
\operatorname{imsup}_{P \in \mathfrak{C}_{n}(\Gamma), P \rightarrow Q} u(P) \leq 0
$$

for any $Q \in \partial \mathfrak{C}_{n}(\Gamma)($ see $[5])$.

The function

$$
\mathbb{P}_{\mathfrak{C}_{n}(\Gamma)}(P, Q)=\frac{\partial \mathbb{G}_{\mathfrak{C}_{n}(\Gamma)}(P, Q)}{\partial n_{Q}}
$$

is called the ordinary Poisson kernel, where $\mathbb{G}_{\mathfrak{C}_{n}(\Gamma)}$ is the Green function.

The Poisson integral of $g$ relative to $\mathfrak{C}_{n}(\Gamma)$ is defined by

$$
\mathbb{P I}_{\mathfrak{C}_{n}(\Gamma)}[g](P)=\frac{1}{c_{n}} \int_{\mathfrak{S}_{n}(\Gamma)} \mathbb{P}_{\mathfrak{C}_{n}(\Gamma)}(P, Q) g(Q) d \sigma
$$


where $g$ is a continuous function on $\partial \mathfrak{C}_{n}(\Gamma)$ and $\frac{\partial}{\partial n_{Q}}$ denotes the differentiation at $Q$ along the inward normal into $\mathfrak{C}_{n}(\Gamma)$.

We set functions $f$ satisfying

$$
\int_{\mathfrak{S}_{n}(\Gamma)} \frac{|f(t, \Phi)|^{p}}{1+t^{\gamma}} d \sigma<\infty
$$

where $p>0$ and

$$
\gamma>\frac{-\aleph^{+}-n+2}{p}+n-1
$$

Further, we denote by $\mathcal{A}_{\Gamma}$ the class of all measurable functions $g(t, \Phi)=(\iota, \ldots,=$ $\left.\left(Y, y_{n}\right) \in \mathfrak{C}_{n}(\Gamma)\right)$ satisfying the following inequality:

$$
\int_{\mathfrak{C}_{n}(\Gamma)} \frac{|g(t, \Phi)|^{p} \varphi}{1+t^{\gamma+1}} d w<\infty,
$$

and the class $\mathcal{B}_{\Gamma}$ consists of all measurable functions $h\left(\iota \cup((t, \Phi))=\left(Y, y_{n}\right) \in \mathfrak{S}_{n}(\Gamma)\right)$ satisfying

$$
\int_{\mathfrak{S}_{n}(\Gamma)} \frac{|h(t, \Phi)|^{p}}{1+t^{\gamma-1}} \frac{\partial \varphi}{\partial n} d \sigma<\infty
$$

We will also consider the class of $\mathrm{a}_{\mathrm{.}}$ ontin ous functions $u(t, \Phi)\left((t, \Phi) \in \overline{\mathfrak{C}_{n}(\Gamma)}\right)$ harmonic in $\mathfrak{C}_{n}(\Gamma)$ with $u^{+}(t, \Phi)-1_{\Gamma}\left(\left(t, \Phi,-\mathfrak{C}_{n}(\Gamma)\right)\right.$, and $u^{+}(t, \Phi) \in \mathcal{B}_{\Gamma}\left((t, \Phi) \in \mathfrak{S}_{n}(\Gamma)\right)$ is denoted by $\mathcal{C}_{\Gamma}$.

Remark 3 If we deno $\Gamma=S_{1}^{+}$jn (1.6) and (1.7), we have

$$
\left.\int_{T_{n}} y_{n}\left|f\left(Y, y_{\eta}\right)\right| y^{n+2}\right)^{-1} d Q<\infty \text { and } \int_{\partial T_{n}}|g(Y, 0)|\left(1+t^{n}\right)^{-1} d Y<\infty .
$$

R cent Ltiau and Yamada (see [6]) obtained the following result.

The $\mathbf{A}$ A Let $g$ be a measurable function on $\partial T_{n}$ such that

$$
\int_{\partial T_{n}} \frac{|g(Q)|}{1+|Q|^{n}} d Q<\infty
$$

Then the harmonic function $\mathbb{P I}_{T_{n}}[g]$ satisfies $\mathbb{P I}_{T_{n}}[g](P)=o\left(r \sec ^{n-1} \theta_{1}\right)$ as $r \rightarrow \infty$ in $T_{n}$.

Recently Wang and Qiao (see [7]) generalized Theorem A to the conical case.

Theorem B Let $g$ be a continuous function on $\partial \mathfrak{C}_{n}(\Gamma)$ satisfying $(1.5)$ with $p=1$ and $\gamma=$ $-\aleph^{-}+1$. Then

$$
u_{\mathbb{P I}_{\mathfrak{C}_{n}(\Gamma)}[g]}=U_{\mathbb{P I}_{\mathfrak{C}_{n}(\Gamma)}[g \mid]}=0 .
$$




\section{Results}

Our main aim in this paper is to give the least harmonic majorant of a nonnegative subharmonic function on $\mathfrak{C}_{n}(\Gamma)$. For related results, we refer the reader to the papers $[8,9]$.

Theorem 1 If $u$ is a subharmonic function on a domain containing $\overline{\mathfrak{C}_{n}(\Gamma)}, u \geq 0$ on $\mathfrak{C}_{n}(\Gamma)$ and $u^{\prime}=u \mid \partial \mathfrak{C}_{n}(\Gamma)$ (the restriction of $u$ to $\left.\partial \mathfrak{C}_{n}(\Gamma)\right)$ satisfies (1.5), then the limit $\mathfrak{u}_{u}(0 \leq$ $\left.u_{u} \leq+\infty\right)$ exists. Further, if $u_{u}<+\infty$, then

$$
u(P) \leq h_{u}(P)=\mathbb{P I}_{\mathfrak{C}_{n}(\Gamma)}\left[u^{\prime}\right](P)+M u_{u} r^{\aleph^{+}} \varphi(\Theta) \quad\left(P=(r, \Theta) \in \mathfrak{C}_{n}(\Gamma)\right),
$$

where $h_{u}(P)$ is the least harmonic majorant of $u$ on $\mathfrak{C}_{n}(\Gamma)$.

\section{Main lemmas}

Lemma 1 Let $u$ be a function subharmonic on $\mathfrak{C}_{n}(\Gamma)$ satisfying $(1.4)_{J} \cdot n y \quad Q \in \mathcal{J}_{\mathcal{C}}(\Gamma)$.

Then the limit $u_{u}\left(-\infty<u_{u} \leq+\infty\right)$ exists.

Proof It suffices to prove that the $\operatorname{limit}_{\lim _{r \rightarrow 0}} r^{\gamma-1} \mathcal{N}_{u}(r)$ exists,

$$
u^{\prime \prime}(r, \Theta)=r^{2-n}(u \circ K)(r, \Theta)
$$

where $K:(r, \Theta) \rightarrow\left(r^{-1}, \Theta\right)$ is the Kelvin trans ee [10], pp.36-37). Consider the auxiliary function

$$
I(s)=s^{\frac{\aleph^{+}}{\chi}} \mathcal{N}_{u}\left(s^{-\frac{1}{\chi}}\right)
$$

on $\left(a^{-\chi},+\infty\right)$. Then, from Re man 1 and $2, I(s)$ is a convex function on $\left(a^{-\chi},+\infty\right)$. Hence

$$
\zeta=\lim _{s \rightarrow \infty} s^{-1} I(s)=\left(n r^{\gamma-1} \mathcal{N}_{i}(r) \quad(-\infty<\zeta \leq+\infty)\right.
$$

exists.

Lemm $2 \perp t u$ be $x$ nonnegative subharmonic function on $\mathfrak{C}_{n}(\Gamma)$ satisfying (1.4) for any $Q \in \mathfrak{a} \mathfrak{C}_{n}$, anw

$+<+\infty$

T) $2 n$

$$
u(r, \Theta) \leq M u_{u^{+}} r^{\aleph^{+}} \varphi(\Theta)
$$

for any $(r, \Theta) \in \mathfrak{C}_{n}(\Gamma)$, where $M$ is a positive constant.

Proof Take any $(r, \Theta) \in \mathfrak{C}_{n}(\Gamma)$ and any pair of numbers $R_{1}, R_{2}\left(0<2 R_{1}<r<\frac{1}{2} R_{2}<+\infty\right)$. We define a boundary function on $\partial \mathfrak{C}_{n}\left(\Gamma ;\left(R_{1}, R_{2}\right)\right)$ by

$$
v(r, \Theta)= \begin{cases}u\left(R_{i}, \Theta\right) & \text { on }\left\{R_{i}\right\} \times \Gamma(i=1,2) \\ 0 & \text { on }\left[R_{1}, R_{2}\right] \times \partial \Gamma\end{cases}
$$


This is an upper semi-continuous function which is bounded above. If we denote Perron-Wiener-Brelot solution of the Dirichlet problem on $\mathfrak{C}_{n}\left(\Gamma ;\left(R_{1}, R_{2}\right)\right)$ with $v$ by $H_{\nu}\left((r, \Theta) ; \mathfrak{C}_{n}\left(\Gamma ;\left(R_{1}, R_{2}\right)\right)\right)$, then we have

$$
\begin{aligned}
u(r, \Theta) \leq & H_{v}\left((r, \Theta) ; \mathfrak{C}_{n}\left(\Gamma ;\left(R_{1}, R_{2}\right)\right)\right) \\
\leq & \frac{1}{c_{n}} \int_{\Gamma} u^{+}\left(R_{1}, \Theta\right) \frac{\partial \mathbb{G}_{\mathfrak{C}_{n}\left(\Gamma ;\left(R_{1}, R_{2}\right)\right)}\left(\left(R_{1}, \Phi\right),(r, \Theta)\right)}{\partial R} R_{1}^{n-1} d S_{1} \\
& -\frac{1}{c_{n}} \int_{\Gamma} u^{+}\left(R_{2}, \Theta\right) \frac{\partial \mathbb{G}_{\mathfrak{C}_{n}\left(\Gamma ;\left(R_{1}, R_{2}\right)\right)}\left(\left(R_{2}, \Phi\right),(r, \Theta)\right)}{\partial R} R_{2}^{n-1} d S_{1},
\end{aligned}
$$

which gives that

$$
u(r, \Theta) \leq M R_{1}^{\gamma-1} \mathcal{N}_{u^{+}}\left(R_{1}\right) r^{\aleph^{-}} \varphi(\Theta)+M R_{2}^{-\aleph^{+}} \mathcal{N}_{u^{+}}\left(R_{2}\right) r^{\aleph^{+}} \varphi(\Theta) .
$$

As $R_{1} \rightarrow 0$ and $R_{2} \rightarrow+\infty$ in (3.2), we complete the proof by

Lemma 3 Let $g$ be a locally integrable function on $\partial \mathfrak{C}_{n}(\Gamma)$ satisfy $(1.5)$ and $u$ be a subharmonic function on $\mathfrak{C}_{n}(\Gamma)$ satisfying

$$
\limsup _{P \in \mathfrak{C}_{n}(\Gamma), P \rightarrow Q}\left\{u(P)-\mathbb{P I}_{\mathfrak{C}_{n}(\Gamma)}[g](P)\right\} \leq 0
$$

and

$$
\limsup _{P \in \mathfrak{C}_{n}(\Gamma), P \rightarrow Q}\left\{u^{+}(P)-\mathbb{P} \mathbb{I}_{\mathfrak{C}}(1, \gamma \mid](P)\right\} \geqslant 0
$$

for any $Q \in \partial \mathfrak{C}_{n}(\Gamma)$. Tl $n$ the limits $u_{u}$ and $u_{u^{+}}\left(-\infty<u_{u} \leq+\infty, 0 \leq u_{u^{+}} \leq+\infty\right)$ exist, and if (3.1) is satisfied,

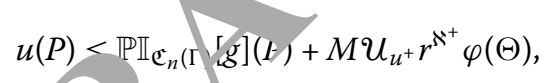

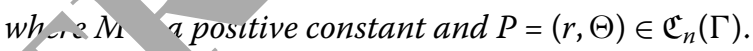

Proc, onsider two subharmonic functions

$$
U(P)=u(P)-\mathbb{P I}_{\mathfrak{C}_{n}(\Gamma)}[g](P) \quad \text { and } \quad U^{\prime}(P)=u^{+}(P)-\mathbb{P I}_{\mathfrak{C}_{n}(\Gamma)}[|g|](P)
$$

on $\mathfrak{C}_{n}(\Gamma)$. From (3.3) and (3.4) we have

$$
\limsup _{P \in \mathfrak{C}_{n}(\Gamma), P \rightarrow Q} U(P) \leq 0 \quad \text { and } \quad \limsup _{P \in \mathfrak{C}_{n}(\Gamma), P \rightarrow Q} U^{\prime}(P) \leq 0
$$

for any $Q \in \partial \mathfrak{C}_{n}(\Gamma)$. Hence it follows from Lemma 1 that the limits $U_{U}$ and $U_{U^{\prime}}(-\infty<$ $\left.u_{U} \leq+\infty, 0 \leq \mathcal{U}_{U^{\prime}} \leq+\infty\right)$ exist. Since

$$
\mathcal{N}_{U}(r)=\mathcal{N}_{u}(r)-\mathcal{N}_{\mathbb{P I}_{\mathfrak{C}_{n}(\Gamma)}[g]}(r) \quad \text { and } \quad \mathcal{N}_{U^{\prime}}(r)=\mathcal{N}_{u^{+}}(r)-\mathcal{N}_{\mathbb{P I}_{\mathfrak{C}_{n}(\Gamma)}[[g]]}(r)
$$


Theorem $\mathrm{B}$ (Theorem 1 will be proved in the next section) gives the existences of the limits $u_{u}, u_{u^{+}}$

$$
u_{U}=u_{u} \quad \text { and } \quad u_{U^{\prime}}=u_{u^{+}} .
$$

Since $0 \leq U^{+}(P) \leq u^{+}(P)+\left(\mathbb{P I}_{\mathfrak{C}_{n}(\Gamma)}[g]\right)^{-}(P)$ on $\mathfrak{C}_{n}(\Gamma)$, it also follows from Theorem B and (3.1) that

$$
u_{U^{+}} \leq u_{u^{+}}<\infty
$$

Hence, by applying Lemma 2 to $U(P)$, we obtain the conclusion from (3.6).

Lemma 4 Let $g$ be a nonnegative lower semi-continuous function on $\partial \mathfrak{C}_{n}(\Gamma) s a_{\text {. }}$.ing (1.5) and $u$ be a nonnegative subharmonic function on $\mathfrak{C}_{n}(\Gamma)$ such that

$$
\limsup _{P \in \mathfrak{C}_{n}(\Gamma), P \rightarrow Q} u(P) \leq g(Q)
$$

for any $Q \in \partial \mathfrak{C}_{n}(\Gamma)$. Then the limit $u_{u}\left(0 \leq U_{u} \leq+\infty\right) \epsilon$,

$$
u(P) \leq \mathbb{P I}_{\mathfrak{C}_{n}(\Gamma)}[g](P)+M u_{u} r^{\aleph^{+}} \varphi(\Theta)
$$

for any $P=(r, \Theta) \in \mathfrak{C}_{n}(\Gamma)$.

Proof Since $-g$ is an upper semi-cont. rus fu action $\partial \mathfrak{C}_{n}(\Gamma)$, it follows from [11], p.3, that

$$
\liminf _{P \in \mathfrak{C}_{n}(\Gamma), P \rightarrow Q} \mathbb{P I}_{\mathfrak{C}_{n}(\Gamma)}[g](P, \geq g
$$

for any $Q \in \partial \mathfrak{C}_{n}(\Gamma)$. W see from (3.7) and (3.8) that

for an $\in \in(\Gamma)$, which gives (3.3). Since $g$ and $u$ are nonnegative, (3.4) also holds. Thus $\mathrm{w}$ obtain conclusion from Lemma 3.

Lem. 5 Let $u$ be subharmonic on a domain containing $\overline{\mathfrak{C}_{n}(\Gamma)}$ such that $u^{\prime}=u \mid \partial \mathfrak{C}_{n}(\Gamma)$ satisfies $(1.5)$ and $u \geq 0$ on $\mathfrak{C}_{n}(\Gamma)$. Then $\mathbb{P I}_{\mathfrak{C}_{n}(\Gamma)}\left[u^{\prime}\right](P) \leq h(P)$ on $\mathfrak{C}_{n}(\Gamma)$, where $h(P)$ is any hirmonic majorant of $u$ on $\mathfrak{C}_{n}(\Gamma)$.

Proof Take any $P^{\prime}=\left(r^{\prime}, \Theta^{\prime}\right) \in \mathfrak{C}_{n}(\Gamma)$. Let $\epsilon$ be any positive number. In the same way as in the proof of Lemma 2, we can choose $R$ such that

$$
\frac{1}{c_{n}} \int_{\mathfrak{S}_{n}(\Gamma ;(R, \infty))} \mathbb{P}_{\mathfrak{C}_{n}(\Gamma)}\left(P^{\prime}, Q\right) u^{\prime}(Q) d \sigma<\frac{\epsilon}{2} .
$$

Further, take an integer $j(j>R)$ such that

$$
\frac{1}{c_{n}} \int_{\mathfrak{S}_{n}(\Gamma ;(0, R))} \frac{\partial \Gamma_{j}\left(P^{\prime}, Q\right)}{\partial n_{Q}} u^{\prime}(Q) d \sigma<\frac{\epsilon}{2} .
$$


Since

$$
\frac{1}{c_{n}} \int_{\mathfrak{S}_{n}(\Gamma ;(0, R))} \frac{\partial \mathbb{G}_{\mathfrak{C}_{n}(\Gamma ;(0, j))}(P, Q)}{\partial n_{Q}} u^{\prime}(Q) d \sigma \leq H_{u}\left(P ; \mathfrak{C}_{n}(\Gamma ;(0, j))\right)
$$

for any $P \in \mathfrak{C}_{n}(\Gamma ;(0, j))$, we have from (3.9) and (3.10) that (see [12])

$$
\begin{aligned}
& \mathbb{P I}_{\mathfrak{C}_{n}(\Gamma)}\left[u^{\prime}\right]\left(P^{\prime}\right)-H_{u}\left(P^{\prime} ; \mathfrak{C}_{n}(\Gamma ;(0, j))\right) \\
& \leq \frac{1}{c_{n}} \int_{\mathfrak{S}_{n}(\Gamma ;(0, R))} \frac{\partial \Gamma_{j}\left(P^{\prime}, Q\right)}{\partial n_{Q}} u^{\prime}(Q) d \sigma \\
& \quad+\frac{1}{c_{n}} \int_{\mathfrak{S}_{n}(\Gamma ;(R, \infty))} \mathbb{P}_{\mathfrak{C}_{n}(\Gamma)}\left(P^{\prime}, Q\right) u^{\prime}(Q) d \sigma \\
& <\epsilon .
\end{aligned}
$$

Here note that $H_{u}\left(P ; \mathfrak{C}_{n}(\Gamma ;(0, j))\right)$ is the least harmonic majora $u$ on $\left.\mathfrak{C}_{n} \Gamma_{\Gamma} ;(0, j)\right)$ (see [13], Theorem 3.15). If $h$ is a harmonic majorant of $u$ on $\mathfrak{C}_{n}\left(I_{1}\right.$, the

$$
H_{u}\left(P^{\prime} ; \mathfrak{C}_{n}(\Gamma ;(0, j))\right) \leq h\left(P^{\prime}\right)
$$

Thus we obtain from (3.11) that

$$
\mathbb{P I}_{\mathfrak{C}_{n}(\Gamma)}\left[u^{\prime}\right]\left(P^{\prime}\right)<h\left(P^{\prime}\right)+\epsilon
$$

which gives the conclusion of Lfmina s

\section{Proof of Theorem 1}

Let $P=(r, \Theta)$ be any point of $\mathfrak{C}_{n}(\Gamma)$ and $\epsilon$ be any positive number. By the VitaliCarathéodory theoren ee [10], p.56), we can find a lower semi-continuous function $g^{\prime}(Q)$ on $\partial \mathfrak{C}_{n}(\Gamma)$ h that

$$
u^{\prime}(2)-?^{\prime}(Q) \text { on } \mathfrak{C}_{n}(\Gamma)
$$

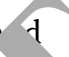

$$
\mathbb{1}, \mathfrak{C}_{n}(\Gamma)\left[g^{\prime}\right](P)<\mathbb{P I}_{\mathfrak{C}_{n}(\Gamma)}\left[u^{\prime}\right](P)+\epsilon
$$

Since

$$
\lim _{P \in \mathfrak{C}_{n}(\Gamma), P \rightarrow Q} u(P) \leq u^{\prime}(Q) \leq g^{\prime}(Q)
$$

for any $Q \in \partial \mathfrak{C}_{n}(\Gamma)$ from (4.1), it follows from Lemma 4 that the limit $U_{u}$ exists (see [11]), and if $\mathcal{U}_{u}<+\infty$, then

$$
u(P) \leq \mathbb{P I}_{\mathfrak{C}_{n}(\Gamma)}\left[g^{\prime}\right](P)+M u_{u} r^{\aleph^{+}} \varphi(\Theta)
$$

Hence we have from (4.2) and (4.3) that (2.1) holds. 
Next we shall assume that $h_{u}(P)$ is the least harmonic majorant of $u$ on $\mathfrak{C}_{n}(\Gamma)$. Set $h^{\prime \prime}(P)$ is a harmonic function on $\mathfrak{C}_{n}(\Gamma)$ such that

$$
u(P) \leq h^{\prime \prime}(P) \quad \text { on } \mathfrak{C}_{n}(\Gamma)
$$

Consider the harmonic function

$$
h^{*}(P)=h_{u}(P)-h^{\prime \prime}(P) \quad \text { on } \mathfrak{C}_{n}(\Gamma)
$$

Since

$$
h^{*}(P) \leq h_{u}(P) \quad \text { on } \mathfrak{C}_{n}(\Gamma)
$$

Theorem B gives that $U_{h^{*+}}<+\infty$. Further, from Lemma 2 we see hat

$$
\limsup _{P \in \mathfrak{C}_{n}(\Gamma), P \rightarrow Q} h^{*}(P)=\limsup _{P \in \mathfrak{C}_{n}(\Gamma), P \rightarrow Q}\left\{\mathbb{P} \mathbb{I}_{\mathfrak{C}_{n}(\Gamma)}\left[u^{\prime}\right](P)-h^{\prime \prime}(P)\right\} \leq
$$

for any $Q \in \partial \mathfrak{C}_{n}(\Gamma)$. From Theorem B and (4.4) we know

$$
u_{h^{*}}=u_{h_{u}}-u_{h^{\prime \prime}}=u_{u}-u_{h^{\prime \prime}} \leq u_{u}-v_{u}=
$$

We see from Lemma 2 that $h^{*}(P) \leq 0$ on $\mathfrak{C}_{h} /$, which shows that $h_{u}(P)$ is the least harmonic majorant of $u(P)$ on $\mathfrak{C}_{\eta}(\Gamma)$. voren 1 is proved.

\section{Conclusions}

In this article, we have sbtained a n-w type of boundary integral behaviors of harmonic functions in a smooth one. As an application, we also gave the least harmonic majorant of a nonnegative subhal function.

\section{Ethics - orovil and consent to participate}

Not ar rical a

\section{onsent ror publication}

Not à ricable.

\section{List of abbreviations}

Not applicable.

\section{Availability of data and materials}

Not applicable.

Competing interests

The authors declare that they have no competing interests.

Authors' contributions

GX drafted the manuscript. MH helped to draft the manuscript and revised the written English. JS helped to draft the manuscript and revised it according to the referee reports. All authors read and approved the final manuscript. 


\section{Author details}

${ }^{1}$ College of Mathematics and Econometrics, Hunan University, Changsha, 410082, China. ${ }^{2}$ Department of Computer Science and Technology, Harbin Engineering University, Harbin, 150001, China. ${ }^{3}$ School of Mechanical Engineering, Taizhou University, Taizhou, 317000, China.

\section{Acknowledgements}

The authors would like to thank the editor, the associate editor and the anonymous referees for their careful reading and constructive comments which have helped us to significantly improve the presentation of the paper.

\section{Publisher's Note}

Springer Nature remains neutral with regard to jurisdictional claims in published maps and institutional affiliations.

Received: 17 November 2016 Accepted: 27 April 2017 Published online: 11 May 2017

\section{References}

1. Rosenblum, G, Solomyak, M, Shubin, M: Spectral Theory of Differential Operators. VINITI, Moscow (1039)

2. Miranda, C: Partial Differential Equations of Elliptic Type. Springer, Berlin (1970)

3. Courant, R, Hilbert, D: Methods of Mathematical Physics, vol. 1. Interscience, New York (1953)

4. Xu, G, Yang, P, Zhao, T: Dirichlet problems of harmonic functions. Bound. Value Probl. 2013, ? (2013)

5. Wanby, G: Convexity of means and growth of certain subharmonic functions in an $n$-direns 21, 29-43 (1983)

6. Zhao, T, Yamada, A Jr.: Growth properties of Green-Sch potentials at infinity. Bound Probl. 20 , 245 (2014)

7. Wang, F, Qiao, L: The w-weak global dimension of commutative rings. Bull. Kore Mat Soc. 52(4), 1327-1338 (2015)

8. Albanese, G, Rigoli, M: Lichnerowicz-type equations on complete manifolds. Ad n' al. 5(3), 223-250 (2016)

9. Fonda, A, Garrione, M, Gidoni, P: Periodic perturbations of Hamiltonian systems. Aa onlinear Anal. 5(4), 367-382 (2016)

10. Helms, LL: Introduction to Potential Theory. Wiley-Interscience, New Yorh

11. Huang, J: Persistent excitation in a shunt DC motor under adaptive contro Astan,. Control 9(1), 37-44 (2007)

12. Feng, C, Huang, J: Almost periodic solutions of nonautonomous Lotka-Volt rra competitive systems with dominated delays. Int. J. Biomath. 8(2), 1550019 (2015)

13. Hayman, WH, Kennedy, PB: Subharmonic Functions, vo 1. Acac c Press, London (1976)

\section{Submit your manuscript to a SpringerOpen ${ }^{\odot}$ journal and benefit from:}

- Convenient online submission

- Rigorous peer review

- Immediate publication on acceptance

- Open access: articles freely available online

- High visibility within the field

Retaining the copyright to your article 